\title{
MOVING TARGET RELATIVE SPEED ESTIMATION IN THE PRESENCE OF STRONG STATIONARY SURROUNDING USING A SINGLE ANTENNA UWB SAR SYSTEM
}

\author{
T.K. Sjögren, V.T. Vu, M.I. Pettersson \\ Blekinge Institute of Technology (BTH), Department of Signal Processing \\ P.O. Box 520, SE-372 25 Ronneby, Sweden \\ E-mail: thomas.sjogren@bth.se, Fax:+46 457 27914, Phone: +46 733384788
}

\begin{abstract}
This paper investigates how relative speed estimation of moving targets in Synthetic Aperture Radar (SAR) images is affected by strong stationary surrounding clutter. The result extends previous work by estimating moving target relative speed under the influence of clutter and Gaussian noise. The results obtained show that good estimates can be obtained even in situations with relatively high clutter and noise, i.e. SNR and SCR lower than 10dB.
\end{abstract}

Index Terms - Synthetic Aperture Radar, Speed Estimation, Ultra Wide Band, Moving Targets

\section{INTRODUCTION}

In the area of remote sensing it is of great interest to image the earth from air- or space based sensors. In response to this, a wide variety of sensors have been developed and used on space- and airborne missions. One of these is radar, which has the unique capability to form remote sensing images independent of weather conditions and daylight. The fact that the radiating source is extremely well known in space and time is a strong advantage of radar compared to optical and other passive sensors. The known source gives radar is unique capabilities to detect changes and motion, such as glacier drift to moving target detection. Also, Synthetic Aperture Radar (SAR) is a very strong remote sensing instrument due to its ability to form images with very high resolution at any range.

There are some problems when imaging with SAR, one is to handle the motion compensation needed for Ultra Wide Band (UWB) systems. This problem grows with increasing integration angle and for airborne systems this is normally more critical than for spaceborne systems. To minimize the errors in motion compensation for UWB SAR and to obtain a focused image, time domain backprojection algorithms are

\footnotetext{
${ }^{1}$ The Authors would like to thank the KK-Foundation for making this research possible, and the support from Swedish Defence Research Agency, Saab Bofors Dynamics, Saab Microwave Systems and Saab Space.
}

best suited [1]. In this research Global Backprojection [2] (GBP) is applied, which is the time domain algorithm that has highest computational cost but also highest accuracy. However, the results of this work are valid with other algorithms. Another effect associated with SAR is that when a moving target is present, the target will be displaced and smeared. Depending on integration time and the relative speed between target and platform, the smearing effect of the target will be more or less severe. However, this effect can be used to detect and to estimate the target properties. To focus a moving target many different approaches have been suggested. If the target is moving with constant speed during the integration time, the target can be focused if a new SAR image is processed using the targets normalized relative speed (NRS) [3]. In [4] an iterative procedure for focusing moving targets based on NRS estimates was presented and tested on data from the CARABAS-II system having a moving target at sea. Even though this system is a low frequency UWB SAR system having long integration time, results were promising, with good focus obtained after two iterations.

This paper presents a study how NRS estimation accuracy is affected by the presence of strong stationary surrounding scatterers. The study consists in simulations of a SAR system according to the specifications of the CARABAS-II SAR, relating to the good experimental results found in [4-5]. However in [4-5] the target was found in an area with low clutter backscatter. This work therefore also relates to the existing low frequency systems at UHF. The lower frequency (VHF, UHF) bands are chosen, due to their strong ability to penetrate vegetation. The reason why the influence of stationary targets is concerned in this paper is that when using the long wavelengths and UWB systems, the background is low and most stationary objects act as very strong point targets. The effect of added white Gaussian noise in the SAR image is also investigated. The effect of the noise should illustrate the behavior of the estimator in microwave SAR where the clutter behaves similar to noise. 


\section{SPEED ESTIMATION FOR MOVING TARGETS IN SYNTHETIC APERTURE RADAR}

When estimating speed for moving targets, different approaches can be undertaken. These approaches can mainly be divided into two groups, phase based or amplitude based. The phase based approaches estimate the phase of the moving target in the raw data or in the SAR image. Normally, the phase chirp rate in azimuth is used for estimating the speed of the target [6-10]. The amplitude based approaches also considers the phase, however they focus the target for a set of speeds [5,11]. These algorithms are both used in the detection and the estimation phase. The speed which gives best focus is the most likely speed. The phase based approach has the advantage that for obtaining an estimate of the moving target speed, no SAR processing is needed and by that has a lower computational cost. The latter approach has the advantage that it focuses all energy and therefore increases the signal to clutter ratio. A third approach for estimating the motion parameters of a moving target is to create a set of SAR images and for each image estimate the image coordinates of the moving target. The set of estimated positions is used for estimating the speed of the moving target [12].

For estimating the chirp rate in a SAR image, which is needed for the phase based approaches; one can apply mainly two different methods. The first method is based on transforming the complex signal containing the moving target into a time-frequency domain by use of e.g. WignerVille transform or the ambiguity function. Then, the Hough transform is used in order to obtain the estimate of the speed. The other method is to apply a phase unwrap on the signal containing the moving target and then apply the finite difference operator twice. After this apply for instance the Gauss Markov estimator as proposed in [4]. This last method is used in this paper and its core can be described by Equation 1 and Equation 2 [4].

$$
\begin{gathered}
\hat{\alpha}=\frac{\mathbf{1}^{\mathrm{T}} \mathbf{C}^{-1} \boldsymbol{\Delta}_{x}^{2} \boldsymbol{\Phi}_{m}(n)}{\mathbf{1}^{\mathrm{T}} \mathbf{C}^{-1} \mathbf{1}} \\
\hat{\gamma}_{t}=\left(\frac{1}{\gamma_{p}^{2}}-\frac{4 \pi}{\lambda_{\mathrm{c}}} \frac{1}{2 r_{t}\left(t_{s t}\right)} \frac{1}{\hat{\alpha}}\right)^{-\frac{1}{2}}
\end{gathered}
$$

Where $\alpha$ is the chirp rate in azimuth of the moving target in the SAR image, $\gamma_{p}$ is the NRS used when SAR processing and $\gamma_{\mathrm{t}}$ is the NRS of the moving target. $\mathbf{C}$ is the covariance matrix of the clutter and noise and $\Delta_{\mathrm{x}}{ }^{2} \boldsymbol{\Phi}_{\mathrm{m}}(\mathrm{n})$ is the second derivative of the measured phase, where the phase is measured as explained in [4]. The centre wavelength is $\lambda_{\mathrm{c}}$ and $r_{t}\left(t_{s t}\right)$ is the true range to the target at time of stationary phase.

\section{MOVING TARGET FOCUSING USING NRS}

As the SAR sensor moves along a synthetic aperture, the range to any coordinate on ground is given by

$$
r(\xi, \eta, t)=\sqrt{\left(v_{p l} t-\xi(t)\right)^{2}+\eta(t)^{2}+h^{2}}
$$

Where $\xi$ and $\eta$ are the ground coordinates in the alongtrack direction and the cross-track direction, respectively, $h$ is the flight altitude of the sensor, $t$ is time and $v_{p l}$ is the speed of the SAR platform. Assuming no target accelerations and let $X_{t}$ and $Y_{t}$ denote the coordinates of a focused target having NRS $\gamma_{t}$. The range to the target from the platform can be described by

$$
r_{t}(t)=\sqrt{\gamma_{t}^{2}\left(v_{p l} t-X_{t}\right)^{2}+Y_{t}^{2}}
$$

Where the NRS $\gamma_{t}$, which depends on target speed in along-track $v_{\xi}$ and cross-track $v_{\eta}$, is defined by

$$
\gamma_{t}=\sqrt{\frac{\left(v_{p l}-v_{\xi}\right)^{2}+v_{\eta}^{2}}{v_{p l}^{2}}}
$$

We now let $X_{p}$ and $Y_{p}$ denote image coordinates for a SAR image processed at the NRS $\gamma_{p}$. The range from the sensor to an arbitrary image coordinate will then be given by

$$
r_{p}\left(X_{p}, Y_{p}, t\right)=\sqrt{\gamma_{p}^{2}\left(v_{p l} t-X_{p}\right)^{2}+Y_{p}^{2}} .
$$

We now see that if $\gamma_{t}$ of the target is known, we can focus the target at $\left(X_{p}, Y_{p}\right)=\left(X_{t}, Y_{t}\right)$ in the SAR image, if we choose $\gamma_{p}=\gamma_{t}$.

\section{SIMULATION EXPERIMENT SETUP}

Two simulation experiments were undertaken, illustrated in Figure 1 and Figure 2. In both experiments, the moving target under interest was distorted by clutter or noise in which the clutter was modeled as a point scatterer and the noise as additive white Gaussian noise. Our signal model for the SAR image in azimuth direction around the target is given in Equation 7.

$$
z(X)=A_{s} \mathrm{e}^{\mathrm{j} 2 \pi\left(\varphi_{0, s}+\frac{\alpha_{s}}{2} X^{2}\right)}+A_{c} \mathrm{e}^{\mathrm{j} 2 \pi\left(\varphi_{0, c}+\frac{\alpha_{c}}{2} X^{2}\right)}+w
$$

Where $X$ is azimuth image coordinate and $w$ is the additive Gaussian noise sequence having variance $\sigma_{n}{ }^{2}$. The amplitude and initial phase of the moving target and stationary scatterer is given by $A_{s}, A_{c}, \varphi_{0, s}$ and $\varphi_{0, c}$. The azimuth chirp rate which we want to estimate using Equation 1 is $\alpha_{s}$, while $\alpha_{c}$ is the chirp rate of the stationary target.

Note that $A_{s}$ and $A_{c}$ will be different for each processing NRS due to the focusing effect [5]. However, for evaluation purposes, we can find these values for each processing speed we want to evaluate.

We define the Signal-to-Noise Ratio (SNR) and Signalto-Clutter Ratio (SCR) according to Equation 8 and Equation 9. From these definitions one easily finds Clutterto-Noise Ratio (CNR). 


$$
\begin{gathered}
S N R=\frac{A_{\mathrm{s}}^{2}}{\sigma_{\mathrm{n}}^{2}} \\
S C R=\frac{R C S_{S}}{R C S_{c}}
\end{gathered}
$$

To find the $\sigma_{n}^{2}$ which correspond to a certain SNR and $A_{s}$, we form the SAR image of only the moving target at the given NRS and choose the peak in the image as the signal amplitude. SCR is simply defined as the ratio between the radar cross sections (RCS) of the moving target and the stationary scatterer.

In SAR image formation, we use Global Backprojection (GBP) with an upsampling factor of 20 and a linear interpolation kernel. Simulation parameters used for both experiments are given in Table 1. They are the same system parameters as used in [4].

In the first experiment the moving target is distorted mainly by the point scatterer which is positioned in the track of the moving target. This is a simple way to model the kind of clutter which characterizes UWB VHF SAR, which means large scatterers that are often well separated and each acts similar to point scatterers. With the system parameters in Table 1, there will be only one point scatterer in the resolution cell that causes this special clutter selection. In this experiment we extend the approach proposed in [4] by estimating the covariance of the stationary scatterer and compare with results achieved without taking the covariance of the stationary scatterer into account.

In the second experiment, the moving target is mainly distorted by the white Gaussian noise. This is to characterize the kind of distortion from clutter which occurs in microwave SAR. The noise is generated as white Gaussian noise in Matlab and for each SNR, 1000 noise distorted images are generated and the estimator is applied for each. From this set of estimates, the variance and the mean is calculated and this is displayed in Figure 3 and Figure 4 .

\begin{tabular}{|l|l|}
\hline Shortest distance to target & $4275 \mathrm{~m}$ \\
\hline Transmission frequency & $20-90 \mathrm{MHz}$ \\
\hline Platform speed & $129 \mathrm{~m} / \mathrm{s}$ \\
\hline Integration time & $160 \mathrm{~s}$ \\
\hline Target speed & $5.4 \mathrm{~m} / \mathrm{s}$, i.e. NRS of 0.958 \\
\hline Processing NRS & 0.93 \\
\hline
\end{tabular}

Table 1. SAR image formation parameters.

\section{SIMULATION RESULTS}

The results obtained for the two simulation experiments are displayed in Figure 3 and Figure 4. In Figure 3 by extending the approach suggested in [4] considering the covariance matrix of the stationary scatterer, we obtain very

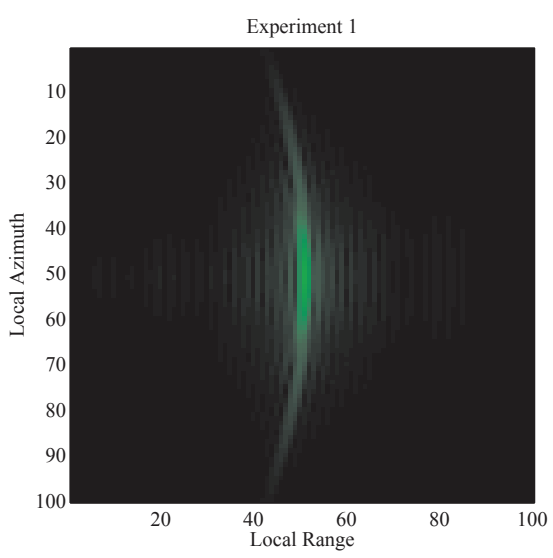

Figure 1. Moving target with defocused stationary target $(\mathrm{SCR}=3 \mathrm{~dB})$.

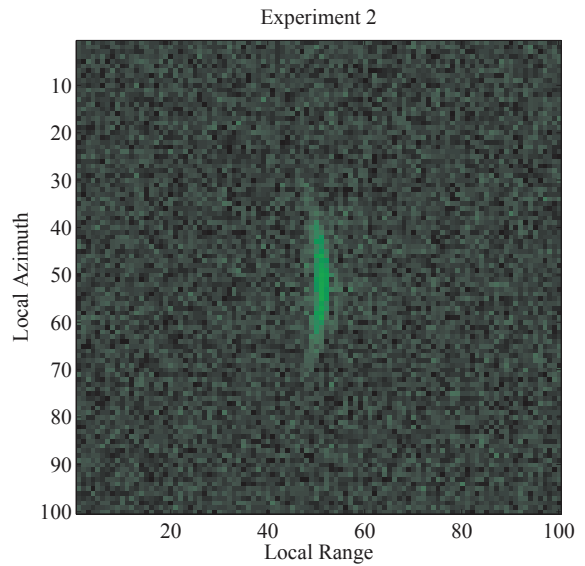

Figure 2. Moving target in Gaussian noise $(\mathrm{SNR}=3 \mathrm{~dB})$.

close to the same results as covariance matrix based on an independent noise background as in [4]. For SCR of approximately $5 \mathrm{~dB}$ and above, error in the mean of the estimate is very low, even below 0.01 in NRS. This corresponds to an error of $1 \mathrm{~m} / \mathrm{s}$ in relative speed. This means that the estimate in the next iteration converge towards the true NRS. One could argue these errors are quite high. However, the estimates in this study are not final estimates, only first iteration estimates and as in [4] there should be further estimates to get the correct speed. Further iterations are not considered here because the purpose of this study is to investigate the effect of strong stationary surrounding upon the first iteration estimates.

In Figure 4 we see that the variance decreases until approximately $7 \mathrm{~dB}$, after which it is very small. However, already at such low SNR as $4 \mathrm{~dB}$, we can see that the mean estimate is good and the variance is about 0.01 NRS, thus allowing for good first iteration estimates. This is a promising result, as it means that if we just detect a target with very low signal level compared to a noisy background, we can be able to get a good first iteration estimate and will be able to converge into a good final NRS estimate. 




Figure 3. Estimated NRS as a function of SCR. The CNR was chosen to $7 \mathrm{~dB}$.

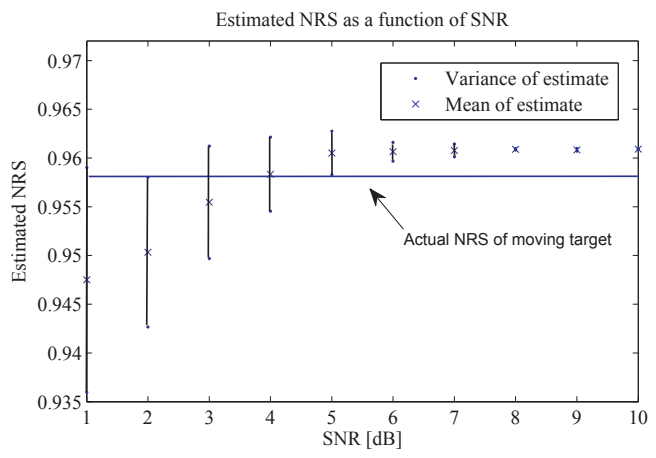

Figure 4. Estimated NRS as a function of SNR. All outliers having estimates over 2 were excluded. This was because the variance of the estimate varied strongly between runs due to outliers. At most 5 estimates in 1000 were excluded.

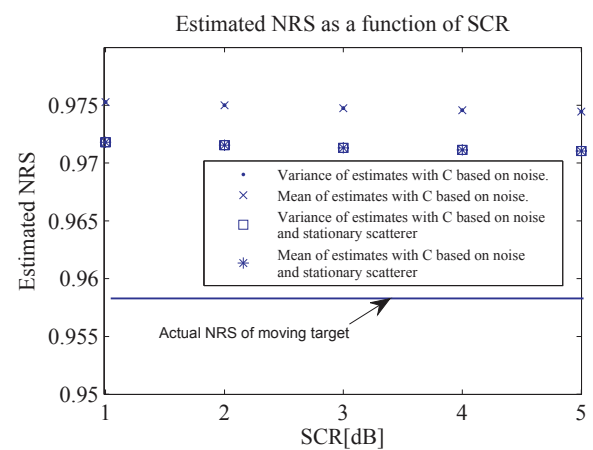

Figure 5. Estimated NRS as a function of SCR, $\gamma_{p}=1$.

A further simulation experiment was made, where the Processing NRS was changed to 1, i.e focusing for a stationary scene. The result is shown in Figure 5. When taking the covariance of the stationary target into account, the first estimate is better compared to when the covariance matrix is only based on noise.

It should further be noted that the estimator has a bias even at high SCR or SNR. It has been found in the simulation experiments that when varying target NRS from a value near processed NRS to values further away from processed NRS, this bias will increase.

\section{CONCLUCIONS AND DISCUSSION}

The work presented in this paper shows that good estimates of moving target relative speed can be obtained by a UWB SAR system. The results can be obtained even when SCR or SNR is relatively low. The threshold is around $4 \mathrm{~dB}$ for SNR and about $5 \mathrm{~dB}$ for $\mathrm{SCR}$ in order to obtain good first iteration estimates. The main contributions of this work is to investigate the behavior of the estimator introduced in [4] and finding estimation thresholds with regard to SCR and SNR as well as extending the approach by estimating the covariance matrix of the clutter. The results show the thresholds are low in both SNR and SCR. A final conclusion is that when taking the clutter into account when estimating the covariance matrix an improvement is obtained when SAR processing for a stationary scene.

\section{REFERENCES}

[1] L.M.H. Ulander, P.-O. Frölind, "Precision processing of CARABAS HF/VHF-band SAR data," Proceedings of IGARSS 1999, Hamburg, Germany, pp. 47-49, 1999.

[2] L.E. Andersson, "On determination of a function from spherical averages," SIAM Journal of Applied Mathematics, pp. 214-341, 1988.

[3] H. Hellsten, L.M.H. Ulander, "Airborne array aperture UWB UHF radar - motivation and system considerations," IEEE Aerospace and Electronic Systems Magazine, pp. 35-45, 2000.

[4] T.K. Sjögren, V.T. Vu, M.I. Pettersson, H.-J. Zepernick, and A. Gustavsson, "Speed estimation experiments for ground moving targets in UWB SAR," Proceedings of IET International Radar Conference, Edinburgh, UK, 2007.

[5] V.T Vu, T.K. Sjögren, M.I. Pettersson, H.-J. Zepernick and A. Gustavsson, "Experimental results on moving targets detection by focusing in UWB low frequency SAR," Proceedings of IET International Radar Conference, Edinburgh, UK, 2007.

[6] F. Zhou, R. Wu, M. Xing, and Z. Bao, "Approach for single channel SAR ground moving target imaging and parameter estimation," IET proceedings in Radar, Sonar and Navigation, pp 59-66, 2007.

[7] S. Barbarossa, A. Farina, "Detection and imaging of moving targets with synthetic aperture radar. 2. Joint time-frequency analysis by Wigner-Ville distribution," IEE Proceedings in Radar and signal processing, pp 89-97, 1992.

[8] S. Barbarossa, "Analysis of multicomponent LFM signals by a combined Wigner-Hough transform," IEEE Transactions on Signal Processing, pp 1511-1515, 1995.

[9] M. Wang, A.K. Chan, C.K. Chui, "Linear frequency-modulated signal detection using Radon-ambiguity transform," IEEE Transactions on Signal Processing, pp. 571-586, 1998.

[10] J.C. Wood, D.T. Barry, "Radon-transformation of timefrequency distributions for analysis of multicomponent signals," IEEE Transactions on Signal Processing, pp. 3166-3177, 1994.

[11] C. H. Gierull, "Ground moving target parameter estimation for two-channel SAR", IEE Proceedings on Radar, Sonar and Navigation, pp 224-233, 2006.

[12] M. Kirscht, "Detection and imaging of arbitrarily moving targets with single-channel SAR", IEE Proceedings on Radar, Sonar and Navigation, pp 7-11, 2003. 